\title{
Frequency characterisation of an optically- interrogated Rogowski coil for smart grid protection applications
}

\author{
Grzegorz Fusiek*, John Nelson*, Philip Orr ${ }^{+}$, Paweł Niewczas*, Campbell Booth* \\ "Institute for Energy and Environment, Department of Electronic and Electrical Engineering, University of Strathclyde, Glasgow \\ G1 1XW, UK, g.fusiek@ strath.ac.uk \\ ${ }^{+}$Synaptec Ltd, Glasgow, UK
}

\begin{abstract}
A preliminary frequency characterization of an optically-interrogated Rogowski coil designed for smart grid protection applications is presented in this paper. The investigation into the sensor performance carried out according to the requirements of the $5 \mathrm{P}$ protection class specified by the IEC 60044-8 standard revealed that the maximum permissible error is breached at the 4th harmonic for the prototype device. It is demonstrated through numerical simulations that by amending a number of parameters, such as the piezoelectric stack capacitance or the Rogowski coil resistance, the sensor performance can be improved and the class requirements readily satisfied. A refined design of the device is proposed accordingly.
\end{abstract}

Keywords- Rogowski coil, optical sensor, piezoelectric transducer, fiber Bragg grating, smart grid protection.

\section{INTRODUCTION}

As opposed to conventional current transformers (CTs), traditional Rogowski coils (RCs) are widely used in electrical power systems for measuring and protection purposes due to their linear response and the ability to measure very large currents without saturation [1], [2]. Because the output voltage of the coil is proportional to the rate of change of the primary current, it must be integrated to recover the measured current, which is usually realized using electronic integrator circuits requiring the electrical power provision at the coil location, and thus limiting their long-distance interrogation. To enable access to multiple, remote, distributed passive current measurements over long distances that can be applicable to a wide range of metering and protection applications, the concept of an optically-interrogated Rogowski coil (ORC) has been proposed by the authors [3]. The ORC formed by connecting an optical low voltage sensor, utilizing a piezoelectric stack transducer and a fibre Bragg grating (FBG) to monitor the Rogowski coil output (Fig. 1), is interrogated remotely by a high-resolution interrogation system (Fig. 2), ensuring that no additional electrical power is required at the ORC location. Preliminary evaluation of the prototype device carried out at a fundamental frequency of $50 \mathrm{~Hz}$ revealed its potential to comply with the requirements of the $5 \mathrm{P}$ protection class specified by IEC 600448 [4].

In this paper, we focus on the frequency characterization of a prototype ORC sensor and demonstrate its capability to comply with relevant IEC standards at harmonics of the fundamental frequency.

\section{OPTICALLY-INTERROGATED ROGOWSKI COIL}

An optically-interrogated Rogowski coil (ORC) utilizing a low voltage piezoelectric transducer to monitor the Rogowski coil output is shown in Fig. 1.

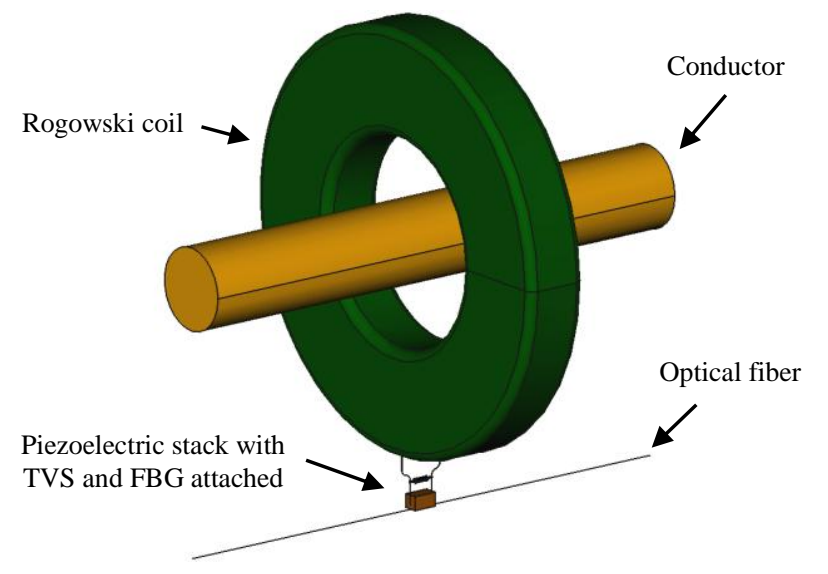

Fig. 1. Hybrid current sensor employing Rogowski coil.

A commercially-available Rogowski coil having a current to voltage ratio of around $1000 \mathrm{~A} / 1 \mathrm{~V}$ at $50 \mathrm{~Hz}$ was used in this study to convert the primary (measured) current into voltage proportional to the rate of change of the primary current according to the equation: $\mathrm{v}(\mathrm{t})=-\mathrm{M} \cdot \mathrm{di}(\mathrm{t}) / \mathrm{dt}$, where $\mathrm{M}=\mathrm{An} \mu_{0}$ is the mutual inductance of the coil, $\mathrm{A}$ is the cross-sectional area of the former, $\mathrm{n}$ is the turns density, and $\mu_{0}$ is the permeability of air [3], [4]. The output voltage of the coil was sensed by a low voltage sensor employing a piezoelectric transducer and a fiber Bragg grating (FBG). A $36 \mathrm{~mm}$ new-generation low voltage stack (P-885.91 PICMA ${ }^{\circledR}$ from Physik Instrumente Ltd (PI) [5]) having a maximum ac operating voltage of $30 \mathrm{~V}_{\mathrm{pk}}$ $\left(21.21 \mathrm{~V}_{\mathrm{rms}}\right)$ was used in this study [4]. To protect the component from depolarization and degradation due to overvoltage conditions, a transient voltage suppression (TVS) diode was connected between the component terminals. A standard $5 \mathrm{~mm}$ long FBG with a central wavelength of $1550 \mathrm{~nm}$ was epoxied to the piezoelectric element using the high temperature adhesive EPOTEK 353ND. The strain induced by an external electric field in the piezoelectric element was converted into a relative change in the FBG peak wavelength. Thus, the primary current measurements can be obtained with a single device and by purely optical interrogation. 


\section{FREQUENCY CHARACTERISATION}

\section{A. Experimental setup}

A diagram of the experimental circuit used for the sensor testing is shown in Fig. 2. As previously [4], a super fluorescent source (SFS) covering the wavelength range $1525-1575 \mathrm{~nm}$ was used to illuminate an FBG sensor. Due to the low voltage output of the coil, a high-resolution optical interrogator utilizing an unbalanced Mach-Zehnder interferometer (MZI) formed between $2 \times 2$ and a $3 \times 3$ single-fusion couplers with a ratiometric output configuration (Fig. 2) was employed in this study [4], [6]-[8]. The optical signals reflected from the sensor were converted to electrical signals by three photodetectors connected to the MZI output ports. The outputs of the photodetectors together with the reference voltage/current were captured by a PXIe data acquisition unit and processed in a PC in order to allow for real-time comparison between the applied primary signal and the optically-interrogated measurement signal. A PXIe-8102 controller and a 16-bit PXIe-6259 data acquisition card (both from National Instruments) were used for generating signals for and acquiring them from the tested devices.

To characterize the ORC device, an ac current source (APTS3 power amplifier, Relay Engineering Services Ltd.) was used to supply primary current to the Rogowski coil and the piezoelectric transducer with an attached FBG was connected to the coil output as a burden. During the device characterization, the current waveforms were obtained from voltage waveforms generated using the PXIe unit and amplified by the amplifier with a gain of $5 \mathrm{~A} / \mathrm{V}$. To monitor primary current and to allow for comparison of the input and output signals of the coil, a shunt resistor was connected to the primary circuit and the voltage across this was used as the reference signal. The resistor was an oil-filled $0.1 \Omega$ resistance standard with a maximum allowed current of $22 \mathrm{~A}$. Due to the shunt resistor current limit, the maximum primary current of $20 \mathrm{~A}$ could be used. To allow the coil to measure currents up to $1 \mathrm{kA}$, 50 turns of primary conductor was wound around the coil crosssection and along its circumference. The coil input and output signals were then acquired by the PXIe unit.

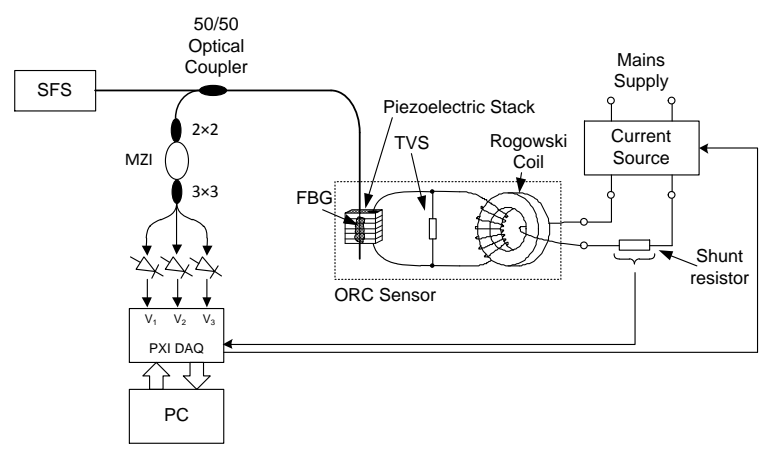

Fig. 2. Circuit diagram for the ORC testing procedure.

A dedicated program created in LabVIEW was used to process the raw sensor response in the form of instantaneous FBG wavelengths and the acquired reference voltage waveforms. Prior to the frequency characterization, the ORC was calibrated at $50 \mathrm{~Hz}$ signals up to $1 \mathrm{kA}$ rated current as described in more detail in [4], [9]. The amplitude and phase errors between the reconstructed and primary currents were calculated as described in [4].

\section{B. Sensor response at harmonic frequencies}

In order to ascertain the performance of the ORC sensor at harmonics of the fundamental frequency, a series of experiments were carried out at frequencies specified by the IEC 60044-8 standard [10], e.g. $20 \mathrm{~Hz}$ (1/3rd harmonic), 100 $\mathrm{Hz}$ (2nd harmonic), $150 \mathrm{~Hz}$ (3rd harmonic), $200 \mathrm{~Hz}$ (4th harmonic), and $250 \mathrm{~Hz}$ (5th harmonic). The accuracy tests were performed with only one single harmonic frequency applied at the primary side for each measurement. The primary current was generated using a combination of the PXIe and the APTS units. The sampling rate for the data acquisition was set to 100 $\mathrm{kS} / \mathrm{s}$. The generation rate was adjusted for each harmonic to ensure the number of samples of $4000 \mathrm{~S} /$ period of the current waveforms. The ORC performance at harmonic frequencies was tested with the sensor calibrated for $50 \mathrm{~Hz}$ signals using the advanced method described in more detail in [4].

Although the ORC response was tested for primary current range up to $1 \mathrm{kA}$ for all the mentioned frequencies, only magnitudes of $10 \%$ of the rated primary current are of interest from the point of view of the protection class standard. Therefore, assuming the ORC rated primary current of $1 \mathrm{kA}$, only amplitude and phase errors calculated for the primary current magnitudes of $100 \mathrm{~A}$ are being considered here. These errors are shown in Fig. 3.

In Fig. 3, the scale on the y axis of the amplitude error plot is set to the maximum permissible amplitude error values at the relevant harmonics. These errors are well below $\pm 2 \%$ for up to 5 th harmonic. The grey area in the phase error plot represents the maximum permissible phase error range at the relevant harmonics. As can be seen, the limit of $\pm 10^{\circ}$ is breached at the $4^{\text {th }}$ harmonic. The phase error is not compensated at frequencies other than $50 \mathrm{~Hz}$ and at $250 \mathrm{~Hz}$ equals to around $14^{\circ}$.
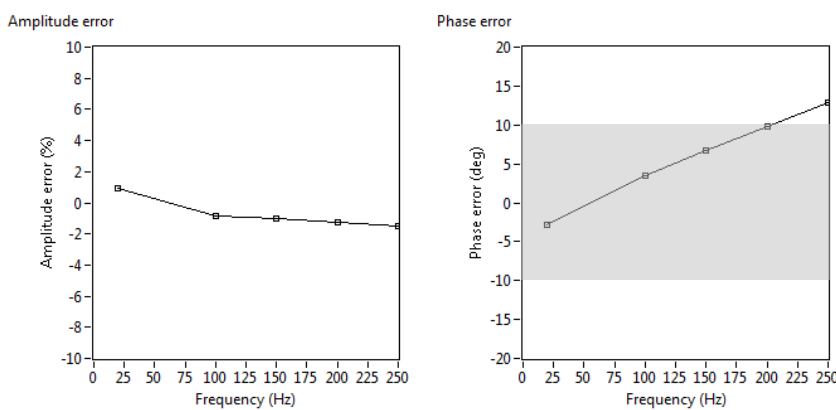

Fig. 3. Amplitude and phase errors for the ORC sensor for different primary currents at room temperature.

\section{ANALYSIS OF THE ORC FREQUENCY RESPONSE}

\section{A. ORC circuit analysis}

The Rogowski coil with a piezoelectric burden forms an RLC circuit as shown in Fig. 4. The piezoelectric transducer is represented by the $\mathrm{RC}$ components, where $R_{p}$ is the stack resistance, and $C$ is its capacitance. The coil is represented by the self-inductance $L_{c}$, the coil resistance $R_{c}$ and its mutual inductance $M$. The coil self-capacitance $C_{S}$ (not shown in the 
figure) is assumed to be negligible in the analysis below when compared with $C$.

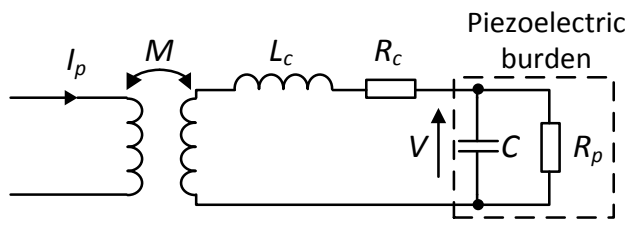

Fig. 4. Optical Rogowski coil (ORC) equivalent circuit.

For the circuit above, the Laplace transfer function describing the relation between the input and output of the system is given by

$$
T F(s)=\frac{1}{s^{2} L C+s\left(R_{c} C+\frac{L}{R_{p}}\right)+1+\frac{R_{c}}{R_{p}}}
$$

For the frequency response analysis, the above function is transferred from the Laplace domain into the complex Fourier domain by substituting $s$ variable with $j \omega$, where $j$ is the complex operator and $\omega$ is the angular frequency. The Bode frequency analysis is then performed and the circuit magnitude and phase response as a function of frequency is obtained.

The separation of the complex frequency response of the system into the magnitude $A(\omega)$ and phase $\varphi(\omega)$ responses is performed using the following equations:

$$
A(\omega)=|T F(\omega)|, \quad \varphi(\omega)=\operatorname{atan}\left[\frac{\operatorname{Imaginary} T F(\omega)}{\operatorname{Real} T F(\omega)}\right]
$$

The two functions when plotted versus frequency form the magnitude and phase Bode plots.

\section{B. Software simulation}

The ORC circuit analysis was performed in a dedicated LabVIEW program using the Control Design and Simulation (CDS) module. The transfer function for the circuit was implemented according to the equations presented above. The program allowed changing the relevant RLC component values, and plotted the Bode magnitude and phase responses for a range of frequencies.

A number of simulations were run for different values of the RLC components to assess how the changes alter the circuit performance. Only frequencies up to 5 th harmonic of a $50-\mathrm{Hz}$ signal $(250 \mathrm{~Hz})$ were considered. Since the amplitude errors achieved during the sensor tests were well below the maximum permissible amplitude error, only Bode phase plots are presented in the follow on discussion and analysis.

As shown in Fig. 5, reduction in the coil resistance significantly affects the phase response of the circuit. The phase displacement decreases with the resistance decrease. The coil self-inductance and stack resistance do not influence the phase response significantly, as shown in Fig. 6 and Fig. 7. The phase shift is influenced significantly by the stack capacitance, as shown in Fig. 8. The phase displacement is larger for larger capacitance.

Based on the circuit analysis presented above, a conclusion may be drawn that by using piezoelectric stacks with lower capacitance and by decreasing the coil resistance the phase displacement can be reduced.
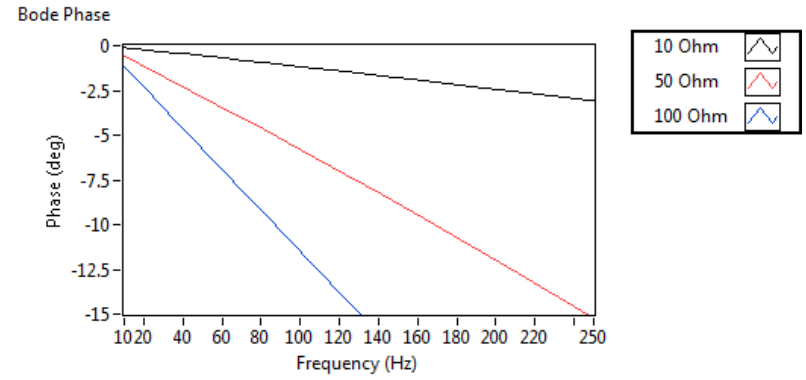

Fig. 5. Bode phase plots for various coil resistance Rc values.

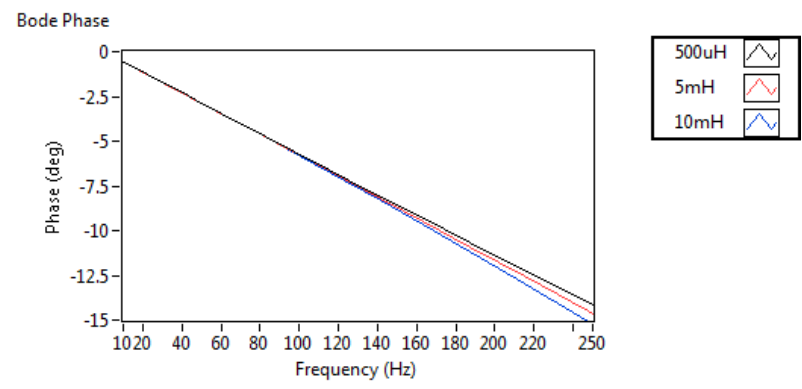

Fig. 6. Bode phase plots for various coil self-inductance $L_{c}$ values.

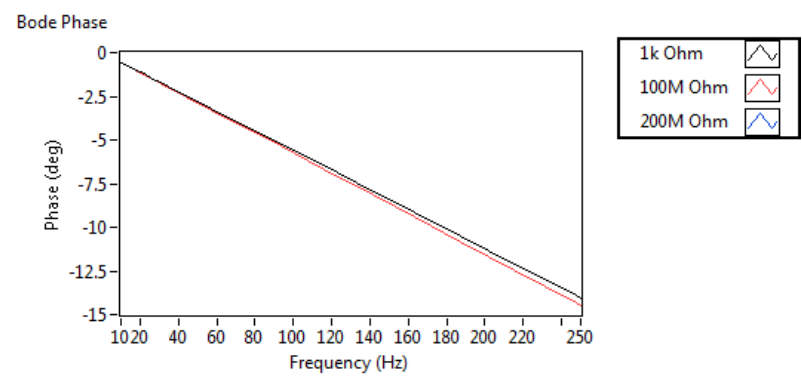

Fig. 7. Bode phase plots for various stack resistance $R_{p}$ values.

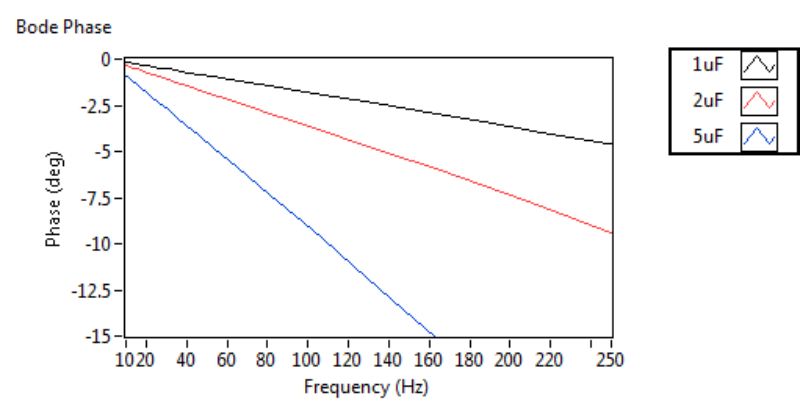

Fig. 8. Bode phase plots for various stack capacitance $C$ values.

\section{ORC REFINED DESIGN}

A simulation of the tested ORC frequency response was performed by using the following component values: $L_{c}=3.14$ $\mathrm{mH}, R_{p}=100 \mathrm{M} \Omega, R_{c}=50 \Omega, C=3.1 \mu \mathrm{F}$. As shown in Fig. 9, the phase displacement of nearly $14^{\circ}$ can be expected at $250 \mathrm{~Hz}$ (5th harmonic), which is in good agreement with the experimental results presented earlier.

Since the piezoelectric stack is formed with a number of layers electrically connected in parallel, each having certain capacitance, the total capacitance of the stack can be reduced 
by limiting the number of layers. The supplier of the piezoelectric transducers also offers stacks shorter than the one used in the presented experiments $(36 \mathrm{~mm})$, exhibiting lower capacitance. For example, the capacitance of a $9-\mathrm{mm}$ stack is only $0.6 \mu \mathrm{F}$ (as opposed to $3.1 \mu \mathrm{F}$ for a $36-\mathrm{mm}$ stack) while the strain to voltage sensitivity remains the same. Furthermore, the Rogowski coil parameters could be amended as well. According to the manufacturer of the coil, by winding it on the same former using a thicker wire, the coil resistance and selfinductance can be reduced down to $17 \Omega$ and $2 \mathrm{mH}$, respectively, while the current to voltage ratio could also be adjusted. The results of the circuit response simulated for the reduced coil resistance and self-inductance assuming that a 9mm stack with lower capacitance is used as the burden are shown in Fig. 9. For such components, the circuit response is significantly improved as the phase displacement of approximately $1^{\circ}$ can be expected at $250 \mathrm{~Hz}$ (5th harmonic). Bode Phase

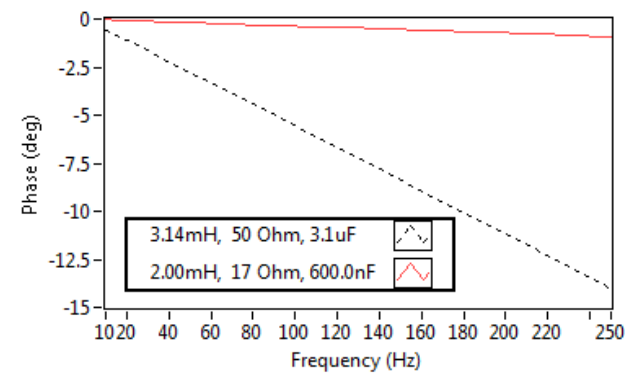

Fig. 9. Bode phase plots for $R_{p}=100 \mathrm{M} \Omega$ and various $L_{c}, R_{c}$ and $\mathrm{C}$ values.

It should be noted that an additional phase displacement between the optical signal and voltage across the piezoelectric burden is introduced due to the hysteresis effect in the piezoelectric element. As demonstrated previously [4], a phase error of $1.3^{\circ}$ was achieved at a voltage of $1 \mathrm{~V}$ corresponding to a rated primary current of $1 \mathrm{kA}$ at room temperature. Since the sensitivity of the phase displacement of $0.5^{\circ} / \mathrm{V}$ was estimated from the experimental results [4], the additional phase error of less than $1^{\circ}$ can be expected at a voltage of $0.1 \mathrm{~V}$ corresponding to a current magnitude of $100 \mathrm{~A}$ at harmonic frequencies $(10 \%$ of the rated primary current according to IEC 60044-8). Therefore, it is envisaged that the total phase error at harmonic frequencies would be well below the permissible $10^{\circ}$ ensuring the sensor conformance with the requirements of the $5 \mathrm{P}$ protection class.

\section{CONCLUSIONS}

In this paper we have reported on the frequency characterization of an optically-interrogated Rogowski coil (ORC) to confirm compliance with the accuracy requirements for protection classes set out by the IEC 60044-8 standard. In the proposed approach, a low-voltage sensor employing a fiber Bragg grating attached to a piezoelectric transducer has been utilized to monitor passively the output of a commercially available Rogowski coil. Preliminary evaluation of the device showed that the amplitude and phase errors at a rated current of $1 \mathrm{kA}$ and at the rated frequency of $50 \mathrm{~Hz}$ are around $0.1 \%$ and $0.1^{\circ}$ respectively when hysteresis compensation is implemented. While the sensor is capable of meeting the $10 \%$ amplitude error requirements at the relevant harmonics $(1 / 3 \mathrm{rd}$, and 2 nd to 5 th harmonics), the $10^{\circ}$ phase error requirements are breached at the 4th harmonic using the initial component selection. Through experiments and numerical simulations, an analysis of the ORC circuit frequency response has been performed and the amendments to the initial design have been proposed. It has been shown that the sensor performance can be improved by reducing the piezoelectric stack capacitance (by reducing stack length), and the resistance of the coil which are the fundamental parameters responsible for phase errors at higher harmonics.

Future work will concentrate on fabrication of the improved ORC device according to the refined design presented in this paper and the evaluation of its performance at higher harmonics and at the operating temperature ranges specified by the IEC 60044-8 standard.

\section{ACKNOWLEDGMENT}

The authors acknowledge the funding and collaboration of Toshiba International (Europe) and Toshiba Corporation. Furthermore, funding from Innovate UK (TSB Project Number 102210) and the UK Engineering and Physical Sciences Research Council (grant reference EP/N508603/1) is acknowledged.

\section{REFERENCES}

[1] "Practical Aspects of Rogowski Coil Applications to Relaying," IEEE PSRC Special Report, IEEE Power Engineering Society, Sep 2010

[2] "IEEE Guide for the Application of Rogowski Coils Used for Protective Relaying Purposes," IEEE Std. C37.235-2007, Feb 2008

[3] P. Orr, P. Niewczas, C. Booth, G. Fusiek, A. Dyśko, F. Kawano, T. Nishida, P. Beaumont, "An Optically-Interrogated Rogowski Coil for Passive, Multiplexable Current Measurement", IEEE Sensors Journal letters, Vol. 13, No 6, June 2013

[4] G. Fusiek, P. Orr, P. Niewczas, A. Dyśko, C.D. Booth, F Kawano, P. Beaumont, G. Baber, T. Nishida, 'Preliminary evaluation of the optically-interrogated Rogowski coil for compliance with power system protection classes', DPSP 2014

[5] Physik Instrumente Ltd. website: http://www.physikinstrumente.com/en/products/prspecs.php?sor tnr $=100810($ Accessed 19/7/2015)

[6] M. D. Todd et al, "A novel Bragg grating sensor interrogation system utilizing a scanning filter, a Mach-Zehnder interferometer and a $3 \times 3$ coupler", Meas. Sci. Technol. 127712001

[7] M. D. Todd, M. Seaver, and F. Bucholtz, "Improved, operationally-passive interferometric demodulation method using $3 \times 3$ coupler," Electronics Letters 38(15):784-786 (2002)

[8] P. Orr and P. Niewczas, "High-Speed, Solid State, Interferometric Interrogator and Multiplexer for Fiber Bragg Grating Sensors," IEEE/OSA Journal of Lightwave Technology 29(22):3387-3392, Nov 2011

[9] G. Fusiek, P. Niewczas, J.R. McDonald, "Improved Method of Hysteresis Compensation for a Piezoelectric Fiber-Optic Voltage Sensor", Optical Engineering, Vol. 46, Issue 3, pp. 034401-1-8, March 2007

[10] IEC, "Instrument transformers - Part 8: Electronic current transformers," First Edition (2002) 